\title{
A SUFFICIENT CONDITION FOR NONVANISHING OF DETERMINANTS
}

\section{P. N. SHIVAKUMAR AND KIM HO CHEW}

ABStract. In this note we derive sufficient conditions for a diagonally dominant reducible matrix to be nonsingular.

1. Throughout this note we are concerned with $A=\left(a_{i j}\right)$, an $n \times n$ matrix which is diagonally dominant and where

$$
J=\left\{i \in N|| a_{i i}\left|>\sum_{j=1 ; j \neq i}^{n}\right| a_{i j} \mid\right\} \neq \varnothing
$$

where $N=\{1,2, \cdots, n\}$. If $J=N, A$ is strictly diagonally dominant and then the Gersgorin circle theorem implies that the determinant of $A$ does not vanish [1, p. 106]. If $A$ is irreducible, Taussky [5] has shown that $A$ is nonsingular. In this note, we prove the following theorem.

THEOREM. Let the matrix $A$ be such that for each $i \notin J$ there is a sequence of nonzero elements of $A$ of the form $a_{i i_{1}}, a_{i_{1} i_{2}}, \cdots, a_{i_{r} j}$ with $j \in J$. Then $A$ is nonsingular.

2. We need the following lemma and results to prove the Theorem.

Lemma 1. Let $A$ satisfy the conditions of the theorem. Then for any nonempty subset $L$ of $N$ such that $L \cap J=\varnothing$, there is a nonzero element $a_{i j}$ with $i \in L$ and $j \notin L$.

Proof. Let $L$ be a nonempty subset of $N$ such that $L \cap J=\varnothing$. Choose $i_{1} \in L$, then $i_{1} \notin J$ and, hence, there is a sequence of nonzero elements of $A$ of the form $a_{i_{1} i_{2}}, a_{i_{2} i_{3}}, \cdots, a_{i_{s-1} i_{s}}$ for some $i_{s} \in J$. Let $r$ be the first integer such that $i_{r} \notin L$ and note that $2 \leqq r \leqq s$ since $i_{1} \in L$ and $i_{s} \notin L$. Then $a_{i_{r-1}} i_{r} \neq 0$ with $i_{r-1} \in L$ and $i_{r} \notin L$. This proves Lemma 1 .

Corollary 2. Let $A$ satisfy the conditions of the Theorem. If $J=\left\{i_{1}, i_{2}, \cdots, i_{k}\right\}$, then there is a permutation $\left(i_{1} i_{2} \cdots i_{n}\right)$ of $N$ such that, for each $j=k+1, \cdots, n, a_{i_{j} i_{l}} \neq 0$ for some $l<j$.

Received by the editors February 22, 1973.

AMS (MOS) subject classifications (1970). Primary 15A15; Secondary 15A15.

Key words and phrases. Matrix, determinant, diagonally dominant, reducible, irreducible, nonsingular, $M$-matrix.

(c) American Mathematical Society 1974 
Proof. If $J=N$, then there is nothing to prove. Suppose $J \neq N$, then $L_{1}=N-J$ is nonempty and $L_{1} \cap J=\varnothing$. Hence, by Lemma 1 , there are an $i_{k+1} \in L_{1}$ and $j \notin L_{1}$ such that $a_{i_{k+1} j} \neq 0$. Since $j \notin L_{1}$, we have $j \in N-L_{1}=J$. Hence $j=i_{l}$ for some $1 \leqq l \leqq k$.

Let $L_{2}=L_{1}-\left\{i_{k+1}\right\}$. If $L_{2}=\varnothing$, then the proof is completed. Suppose $L_{2} \neq \varnothing$. Obviously $L_{2} \cap J=\varnothing$. Again, by Lemma 1 , there are $i_{k+2} \in L_{2}$ and $j \notin L_{2}$ such that $a_{i_{k+2}} \neq 0$. Since $j \notin L_{2}$, we have $j \in J \cup\left\{i_{k+1}\right\}$. Hence $j=i_{l}$ for some $1 \leqq l \leqq k+1$. The corollary is proved by repeating the above process until $L_{p}=L_{p-1}-\left\{i_{k+p-1}\right\}=\varnothing$.

Corollary 3. Let $A$ satisfy the conditions of the Theorem. If $J=$ $\left\{i_{1}, i_{2}, \cdots, i_{k}\right\}$, then there is a permutation $\left(i_{1} i_{2} \cdots i_{n}\right)$ of $N$ such that

are positive.

$$
g_{j}=\left|a_{i_{j} i_{j}}\right|-\sum_{l=j+1}^{n}\left|a_{i_{j} i_{l}}\right|, \quad j=1,2, \cdots, n,
$$

Proof. From Corollary 2, there is a permutation $\left(i_{1} i_{2} \cdots i_{n}\right)$ of $N$ such that, for each $j=k+1, \cdots, n, a_{i_{j} i_{l}} \neq 0$ for some $l<j$. Notice that if $i_{j} \in J$, then

$$
g_{j}=\left|a_{i_{j} i_{j}}\right|-\sum_{l=j+1}^{n}\left|a_{i_{j} i_{l}}\right| \geqq\left|a_{i_{j} i_{j}}\right|-\sum_{l=1 ; l \neq j}^{n}\left|a_{i_{j} i_{l}}\right|>0 .
$$

Hence $g_{j}>0, j=1,2, \cdots, k$. For $j=k+1, \cdots, n$,

$$
\begin{aligned}
g_{j} & =\left|a_{i_{j} i_{j}}\right|-\sum_{l=j+1}^{n}\left|a_{i_{j} i_{l}}\right| \\
& >\left|a_{i_{i} i_{j}}\right|-\sum_{l=j+1}^{n}\left|a_{i_{j} i_{l}}\right|-\sum_{l=1}^{j-1}\left|a_{i_{j} i_{l}}\right| \geqq 0
\end{aligned}
$$

since $a_{i_{j} i_{l}} \neq 0$ for some $l<j$. This completes the proof.

Corollary 4. Let $A$ satisfy the conditions of the Theorem. If $A$ is real and $a_{i j} \leqq 0, a_{j j}>0$, then $A$ is an M-matrix [2].

Proof. Let $J=\left\{i_{1}, i_{2}, \cdots, i_{k}\right\}$. Then it follows from Corollary 3 that there is a permutation $\left(i_{1} i_{2} \cdots i_{n}\right)$ of $N$ such that

$$
g_{j}=\left|a_{i_{j} i_{j}}\right|-\sum_{l=j+1}^{n}\left|a_{i_{j} i_{l}}\right|>0, \quad j=1,2, \cdots, n .
$$

Now, $\operatorname{det} A=g_{1} \operatorname{det} A\left(i_{1}\right)+\operatorname{det} A\left\{i_{1}\right\}$, where $A(i, j, \cdots, k)$ denotes the matrix obtained from $A$ with $i-, j-, \cdots, k$-rows and columns deleted, and $A\left\{i_{j}\right\}$ is the matrix $A$ with the entry $a_{i_{i} i_{j}}$ replaced by $\sum_{l=j+1}^{n}\left|a_{i_{i} i_{l}}\right|$. From [1, p. 294, Problem 9], we have $\operatorname{det} A\left\{i_{1}\right\} \geqq 0$. Thus

$$
\operatorname{det} A \geqq g_{1} \operatorname{det} A\left(i_{1}\right) \text {. }
$$


Similarly, we have

$$
\begin{aligned}
\operatorname{det} A\left(i_{1}\right) \geqq g_{2} \operatorname{det} A\left(i_{1}, i_{2}\right), & \\
\cdot & \cdot \\
& \cdot \\
\operatorname{det} A\left(i_{1}, i_{2}, \cdots, i_{n-1}\right) & \geqq g_{n} .
\end{aligned}
$$

Hence $\operatorname{det} A \geqq \prod_{j=1}^{n} g_{j}>0$.

In the same way, we can show that the determinant of each of the principal submatrix of $A$ is positive, therefore $A$ is an $M$-matrix.

3. Proof of the Theorem. Ky Fan [2] has shown that if a complex matrix $A=\left(a_{i j}\right)$ and an $M$-matrix $B=\left(b_{i j}\right)$ satisfy

$$
b_{i i} \leqq\left|a_{i i}\right| \text { for all } i \in N
$$

and

$$
\left|a_{i j}\right| \leqq\left|b_{i j}\right| \text { for } i \neq j,
$$

then $|\operatorname{det} A| \geqq \operatorname{det} B$. Our Theorem follows immediately, since the real matrix $B=\left(b_{i j}\right)$, given by

$$
\begin{aligned}
b_{i j} & =\left|a_{i j}\right| & & \text { if } i=j, \\
& =-\left|a_{i j}\right| & & \text { if } i \neq j,
\end{aligned}
$$

is an $M$-matrix (by Corollary 4), and in addition to (3.1) and (3.2), it satisfies where the $g_{j}$ are given in (2.1).

$$
\operatorname{det} B \geqq \prod_{j=1}^{n} g_{j}>0,
$$

4. For the case where $J=N$, Ostrowski ([3], [4]) has given the following lower bounds for $|\operatorname{det} A|$ :

$$
M_{1}=\prod_{i=1}^{n}\left(\left|a_{i i}\right|-\sum_{j=i+1}^{n}\left|a_{i j}\right|\right)
$$

and

$$
M_{2}=\prod_{i=1}^{n}\left(\left|a_{i i}\right|-\sum_{j=1}^{i-1}\left|a_{i j}\right|\right) .
$$

Observe that, for $J=N$, the $g_{j}$ given in (2.1) are positive for any arbitrary permutation $\left(i_{1} i_{2} \cdots i_{n}\right)$ of $N$. If we choose the permutation $(12 \cdots n)$, then our lower bound $\prod_{i=1}^{n} g_{i}=M_{1}$. If the permutation is chosen to be $(n(n-1) \cdots 1)$, then $\prod_{i=1}^{n} g_{i}=M_{2}$. The following simple example shows that we have a better lower bound. 
EXAMPLE 1. Let

$$
A=\left(\begin{array}{ccc}
3 & 2 & \frac{1}{2} \\
2 & 5 & 2 \\
\frac{3}{2} & 2 & 4
\end{array}\right)
$$

Then $M_{1}=\frac{1}{2} \cdot 3 \cdot 4=6, M_{2}=3 \cdot 3 \cdot \frac{1}{2}=\frac{9}{2}$ and choosing the permutation (2 31 ), we get

$$
\prod_{i=1}^{3} g_{i}=1 \cdot \frac{5}{2} \cdot 3=\frac{15}{2} .
$$

In general, since for each possible permutation $\left(i_{1} i_{2} \cdots i_{n}\right)$ of $N$ (obtained from Corollary 3 ) we can find a corresponding set of $g_{j}$ 's, and thus we may have more than one lower bound for $|\operatorname{det} A|$.

EXAMPLE 2. Consider the diagonally dominant matrix

$$
A=\left(\begin{array}{lllll}
1 & \frac{1}{2} & 0 & 0 & 0 \\
1 & 2 & 1 & 0 & 0 \\
2 & 2 & 4 & 0 & 0 \\
0 & 3 & 3 & 9 & 3 \\
0 & 0 & 4 & 4 & 8
\end{array}\right)
$$

Clearly $A$ is reducible. Now, $J=\{1\}$ and we have the following sequences of nonzero elements of $A$ :

$$
\left\{a_{21}\right\}, \quad\left\{a_{31}\right\}, \quad\left\{a_{42}, a_{21}\right\}, \quad\left\{a_{53}, a_{31}\right\} .
$$

Hence the matrix $A$ satisfies the conditions of the Theorem.

\section{REFERENCES}

1. R. Bellman, Introduction to matrix analysis, McGraw-Hill, New York, 1960. MR 23 \#A153.

2. Ky Fan, Inequalities for sum of two M-matrices, Inequalities I, Academic Press, New York and London, 1967, pp. 105-117. Edited by Oved Shisha.

3. A. Ostrowski, On some conditions for nonvanishing of determinants, Proc. Amer. Math. Soc. 12 (1961), 268-273. MR 25 \#1168.

4. - Note on bounds for determinants with dominant principal diagonal, Proc. Amer. Math. Soc. 3 (1952), 26-30. MR 14, 611.

5. O. Taussky, A recurring theorem on determinants, Amer. Math. Monthly 56 (1949), 672-676. MR 11, 307.

Department of Mathematics, University of Manitoba, Winnipeg, Manitoba, CANADA R3T 2N2 